\title{
Evidenzbasierung und Zusammenhangsanalysen in der Lehrkräftebildung
}

\section{Diagnosewissen, Selbstkonzept und Einstellungen zu Inklusion in manifesten Wachstumsmodellen}

\author{
Alexander Wedel, Jan Pfetsch \& Angela Ittel
}

Journal für Psychologie, 27(2), 356-381

https://doi.org/10.30820/0942-2285-2019-2-356

www.journal-fuer-psychologie.de

\section{Zusammenfassung}

Die Lehrkräftebildung verwendet das wissenschaftliche Konstrukt der Evidenz als Grundlage von Entscheidungsprozessen in der Gestaltung von Lerngelegenheiten zu inklusivem Unterrichten. Zwar ist der Zusammenhang von Diagnosewissen und positiver Einstellung zu inklusivem Unterrichten anerkannt, die dazu verfügbare quantitative Evidenz unterliegt jedoch zahlreichen Einschränkungen. Wir diskutieren die Bedingungen der Herstellung von Evidenz in der Lehrkräftebildung und zeigen im Kontext von Zusammenhangsanalysen typische Fehlerquellen klassischer Berechnungsverfahren auf. Datengrundlage ist eine quasi-experimentelle Studie mit 63 Lehramtsstudierenden, die problemorientiert oder instruktionsbasiert pädagogisches Diagnostizieren lernen. Durch die Anwendung eines innovativen statistischen Verfahrens, mit dem manifeste Wachstumskurvenmodelle in kleinen Stichproben berechnet werden können, zeigen wir, dass ein Zuwachs an Diagnosewissen mit einer Steigerung positiver Einstellungen zu inklusivem Unterrichten einhergehen kann. Im Anschluss diskutieren wir die Bedeutung der Ergebnisse für den Übergang angehender Lehrkräfte in die Schulpraxis.

Schlüsselwörter: Lehrkräftebildung, Diagnostische Kompetenz, Inklusion, Evidenz, Wachstumskurvenmodell, Korrelation

\section{Summary}

Evidence-based teacher education and correlation analyses. Diagnostic knowledge, self-concept and attitudes towards inclusive teaching in a manifest growth-curve model.

Teacher education utilizes the scientific construct of evidence for decisions on the design of teacher trainings. While the relationship between diagnostic knowledge and positive at- 
titudes towards inclusive teaching is widely accepted, the available evidence is constrained by many factors. We discuss the conditions of constructing evidence in teacher education and show typical errors of classical correlation analyses. Our analyses are based on a quasi-experimental study with 63 pre-service teachers, who learned how to diagnose students either with problem-based learning or with instruction-based learning. Applying an innovative statistical method that allows for calculating growth-curve models with small sample sizes, we show that a growth in diagnostic knowledge can correlate with a growth in positive attitudes towards inclusive teaching. In the discussion we focus on the meaning of the results for the transition of pre-service teachers into teaching.

Key words: teacher education, diagnostic competence, inclusion, evidence, growth-curve model, correlation

\section{$1 \quad$ Einleitung}

Evidenz kann die Lehrkräftebildung über Rahmenbedingungen, Prozesse und Ergebnisse von Bildung informieren, die zum gegenwärtigen Zeitpunkt mit wissenschaftlichen Methoden messbar sind. Mit dem Ziel eines weitgehend inklusiven Schulsystems steigt auch der Bedarf an evidenzbasierten Lerngelegenheiten zur Förderung inklusiver Lehrkompetenzen (Darling-Hammond 2017). Da klassische Kompetenzmodelle Einstellungen als zentrale Komponente professioneller Lehrkompetenz betrachten (Baumert und Kunter 2006), stellen inklusionsbejahende Einstellungen und Möglichkeiten ihrer Förderung einen Schwerpunkt der Forschung zur Lehrkräftebildung dar (Hellmich und Görel 2014).

Positive Einstellungen $\mathrm{zu}$ inklusivem Unterrichten gehören $\mathrm{zu}$ den wichtigsten Einflussfaktoren einer lernförderlichen Unterrichtspraxis in heterogenen Lerngruppen (Avramidis und Norwich 2002; Savage und Erten 2015). Trotz überwiegend positiver Einstellungen berichten viele Lehrkräfte und Lehramtsstudierende jedoch Ängste und Befürchtungen im Bezug auf die Unterrichtsdurchführung (Schwab und Seifert 2015; Syring et al. 2018) und Lehrkräfte geben einen Mangel an inklusionsbezogenen Ausund Weiterbildungsinhalten an (OECD 2014).

Die Forschung zur Lehrkräftebildung knüpft an diese Ergebnisse durch die Untersuchung von Wissensbeständen und Maßnahmen zur Wissensvermittlung an, die eine positive Einstellungsbildung und -änderung bewirken können (Schürer 2018). Dies ist auch der Schwerpunkt der vorliegenden Studie, die den Zusammenhang von kognitiven Fähigkeiten im Bereich des Diagnosewissens mit Einstellungen zu inklusivem Unterrichten in einer quasi-experimentellen Studie mit einem selbstgesteuerten versus instruktionsbasierten hochschuldidaktischem Lernsetting untersucht. Dabei wird 
ein methodischer Weg der Analyse dargestellt, der einen unverzerrten Blick auf diese Zusammenhänge von Diagnosewissen, Einstellungen zu inklusivem Unterrichten und diagnostischem Selbstkonzept sowie deren Veränderung ermöglichen. Kern des vorliegenden Beitrags ist die Frage: Wie entwickeln sich diese Zusammenhänge, wenn Diagnosewissen mit selbstgesteuertem Lernen oder mit instruktionsbasiertem Lernen vermittelt wird?

\section{Theoretischer Rahmen und Forschungsstand: Diagnosewissen und Selbstkonzept als Basis der Einstellung zu inklusivem Unterrichten}

Einstellungen sind evaluative Reaktionen auf Einstellungsobjekte und beinhalten eine kognitive, affektive und behaviorale Komponente (Bohner und Wänke 2002). Einstellungsbildung und -änderung werden zumeist mit dualen Prozessmodellen und insbesondere mit dem Elaboration Likelihood Model (ELM) erklärt (Petty und Cacioppo 1986). Das ELM erklärt Einstellungsänderungen durch das Ausmaß an kognitiver Elaboration, mit der eine Botschaft in Bezug auf das Einstellungsobjekt verarbeitet wird, und die wahrgenommene Stärke, die diese Botschaft hat.

Mehrere Studien weisen darauf hin, dass sowohl wissenschaftliche Evidenz als starkes Argument wahrgenommen wird (Winter und Krämer 2016; Winter et al. 2015) als auch selbstgenerierte Informationen (Darke et al. 1998). So weist Wenden (1998) darauf hin, dass selbstgesteuertes Lernen zur Analyse und Revision bisheriger Einstellungen und Überzeugungen führen kann. Diesen Effekt auf Einstellungsänderungen unterstützen auch aktuellere Studien zu problemorientiertem Lernen. Diese Form selbstgesteuerten Lernens führt im Vergleich zu lehrkräftezentrierten Lernformen eher zu Einstellungsänderungen in Bezug auf die Lerninhalte, was sowohl Meta-Analysen zeigen (Dmirel und Dağyar 2016) als auch Studien spezifisch in Bezug auf Lehramtsstudierende (z. B. Kuvac und Koc 2018).

Im ELM kommt auch dem Selbstkonzept eine Rolle in der Einstellungsänderung zu. Die > functional matching < Hypothese besagt, dass die Verarbeitung von zum Selbstkonzept kongruenten Informationen eine positive Bewertung der Informationen und eine stärkere Einstellungsänderung bewirkt (Lavine und Snyder 1996; Petty und Wegener 1998), insbesondere wenn das Selbstkonzept in der Beurteilungssituation salient ist (Fleming und Petty 2000).

In einem engen konzeptionellen Zusammenhang zu inklusionsorientiertem Unterrichten steht nicht nur das professionelle Selbstkonzept, sondern auch das Wissen aus dem Bereich pädagogischer Diagnostik. So wird Diagnosewissen nicht nur konzeptionell als Bestandteil des Professionswissens angesehen (Voss et al. 2015), sondern in 
neueren Modellen adaptiver Lehrkompetenz auch als Voraussetzung inklusiven Unterrichtens beschrieben (Baumert und Kunter 2011; Rey et al. 2018) und ist darüber hinaus ein zentraler Bestandteil von Testinstrumenten zu inklusionsrelevanten Wissensbeständen (König et al. 2019; Marx et al. 2017).

Die empirischen Befundlage des Zusammenhangs von Diagnosewissen und Einstellungen zu inklusivem Unterrichten wird in systematischen Reviews als sehr einheitlich dargestellt. Sowohl das Meta-Review von Mieghem und anderen (2018), als auch vorherige Reviews zu inklusionsbezogenen Einstellungen von Lehrkräften (Avramidis und Norwich 2002; de Boer, Pijl und Minnaert 2011) berichten einen positiven mittleren Zusammenhang. Grundlage dieser Bewertung sind jedoch Studien mit bedeutsamen methodischen Einschränkungen. Dies betrifft zum einen deskriptive Beobachtungen eines parallelen Anstiegs bzw. Niveaus von Wissen und Einstellungen (Kurniawati et al. 2017; Liu 2016; Mulholland 2016; Youssef und Youssef 2015) und zum anderen Interventionsstudien, die lediglich querschnittliche Zusammenhänge mit Pearson Korrelationen berechnen, nicht aber Zusammenhangsveränderungen schätzen oder für eine autokorrelative Struktur der Konstrukte kontrollieren (Avramidis, Bayliss und Burden 2002; Batsiou et al. 2008; Ghanizadeh, Bahredar und Moeini 2006). So bleibt unklar, ob in einer großen Zahl verfügbarer Studien das Wissen eine Grundlage der Einstellung bildete oder die Einstellung zum Wissenserwerb motivierte. Beide Ansätze der Datenanalyse resultieren größtenteils aus einem Mangel adäquater Stichproben bzw. geeigneter Analyseverfahren für die vorliegenden Stichproben.

Eine weitere Einschränkung ist die Erfassung der Wissensbestände durch Selbsteinschätzungen (Avramidis, Bayliss und Burden 2002; Batsiou et al. 2008; Mulholland, Cumming und Jung 2015; Mulholland 2016; Sermier Dessemontet, Benoit und Bless 2011). Selbsteinschätzungen zu Wissen und Fähigkeiten sind Teil des professionellen Selbstkonzepts und stehen nur in einem mittleren Zusammenhang zum objektiven Professionswissen (Retelsdorf et al. 2014). In einem Review beurteilen Ewing, Monsen und Kielblock (2017) den Zusammenhang zwischen den Einstellungen zu inklusivem Unterrichten und dem professionellen Selbstkonzept, das auch explizit als solches gemessen wurde, als positiv. Das Review differenziert dabei zwar nicht zwischen verschiedenen Facetten des professionellen Selbstkonzepts, jedoch sind die Ergebnisse sehr einheitlich.

Zum Zusammenhang zwischen dem professionellen Selbstkonzept und dem Diagnosewissen ist die Studienlage klarer. So kommen Interventionsstudien mit Lehramtsstudierenden ebenso zum Ergebnis positiver mittlerer bis großer Zusammenhänge (Klug et al. 2013; Wedel et al. 2019), wie auch querschnittlich angelegte Studien (Klug, Bruder und Schmitz 2016; Ohle et al. 2015).

Zusammengefasst besteht nach wie vor Forschungsbedarf zu Zusammenhängen zwischen Diagnosewissen, Selbstkonzept und Einstellungen zu inklusivem Unterrichten. Wenngleich die zahlreichen Interventionsstudien ein Beleg für die Veränderbarkeit 
dieser Konstrukte in formalen Bildungskontexten sind (z. B. Lehrveranstaltungen, Weiterbildungen), fehlt es an belastbaren statistischen Ergebnissen, die mit größerer Sicherheit als wissenschaftliche Evidenz interpretiert werden können. Besonderes Interesse besteht an Interventionsstudien, da diese über die querschnittliche Zusammenhänge hinaus auch die Veränderung dieser Zusammenhänge adressieren und damit Auskunft über die Effektivität von Wissenserwerb als Grundlage von Einstellungsänderungen geben.

\section{$3 \quad$ Herstellung von inklusionsbezogener Evidenz in der Lehrkräftebildung}

In der Wissenschaftstheorie beschreibt Evidenz die regelgeleitete Verbindung theoretischer Wissensbestände mit einem Ausschnitt der Realität (Mullen 2015). Bromme, Prenzel und Jäger (2016) schlagen vor, dass Evidenz mit wissenschaftlichen Methoden gewonnene Daten oder Befunde sind, die zur Prüfung von Theorien verwendet werden. Diese Definition entspricht dem Begriff forschungsbezogener Evidenz im kritischen Rationalismus, dessen Falsifikationsprinzip zu einer bedeutenden Wissens- und Entscheidungsgrundlage in allen gesellschaftlichen Feldern geworden ist.

Die Erzeugung von Evidenz dieser Art ist in Bildungskontexten jedoch häufig problematisch. Spiel und Strohmeier (2012) stellen fest, dass nur selten randomisierte Experimental- und Kontrollgruppen oder ausreichend große Stichproben für geeignete statistische Modelle zur Verfügung stehen. Darüber hinaus ist auf struktureller Ebene, das heißt im System der Lehrkräftebildung, häufig keine Population gegeben, auf die verallgemeinert werden könnte. Dies erschwert die Anwendung probabilistischer Messtheorien und Falsifikationen generell (Bromme, Jäger und Prenzel 2014). Im Kontext inklusiven Unterrichtens spitzt sich diese Konstellation noch weiter zu. Auf bildungspolitischer Ebene existiert zwar die normative Anforderung inklusionsbezogene Kompetenzen zu vermitteln (KMK und HRK 2015), doch existieren weder eine einheitliche Definition von Inklusion, noch direkt vergleichbare Ausbildungsstrukturen und -ziele (Greiten et al. 2017).

Um in dieser methodologisch widersprüchlichen Situation evidenzbasierte Entscheidungen treffen zu können, existieren sowohl konzeptionelle als auch methodische Ansätze. Auf konzeptioneller Ebene wird Evidenz mit dem Begriff der evidenzbasierten Praxis vom Forschungs- auf das Praxissetting ausgeweitet. Dabei wird fallbezogenes Wissen und individuelle Expertise, die aus reflektierten subjektiven Erfahrungen und Routinen besteht, ebenfalls als Evidenz anerkannt (Sackett et al. 1996). Dies eröffnet jedoch noch weitestgehend unbeantwortete Fragen, beispielsweise der Gewichtung von Expertise und wissenschaftlicher Evidenz in Entscheidungsvorgängen. Auf methodi- 
scher Ebene können mit baysianischer Inferenzstatistik die Ergebnisse von Studien aus unterschiedlichen Kontexten verglichen werden (Schum 2012). Die dafür verfügbaren statistischen Methoden stellen jedoch so hohe Anforderungen an methodische Expertise, dass ihre Anwendung auch außerhalb der Lehrkräftebildung vergleichsweise selten erfolgt (Gopnik und Tenenbaum 2007).

Probabilistische Zusammenhangsanalysen sind eine zentrale Grundlage der evidenzbasierten Maßnahmenentwicklung, nicht zuletzt, um Prädiktoren von Einstellungen zu inklusivem Unterrichten zu identifizieren und damit Konzepte professioneller Kompetenz von Lehrkräften zu informieren. Die vorliegende Studie stellt daher das Verfahren der messwiederholten Korrelation und die darauf aufbauende Modellklasse der manifesten Wachstumskurvenmodelle vor. Diese Verfahren können mit wenigen Einschränkungen auch mit kleineren Stichproben zuverlässige Parameterschätzungen in der Analyse von Zusammenhangsveränderungen vornehmen und damit die Bildung von Evidenz im Bereich inklusionsbezogener Kompetenzen unterstützen.

\section{Fragestellung und Hypothesen}

Wie könnte Evidenz über die Vermittlung von Diagnosewissen als Basis inklusionsbejahender Einstellungen beschaffen sein? Hierzu kann eine Aussage aus dem ELM und den Kompetenzmodellen der Lehrkräftebildung abgeleitet werden. Selbstgesteuertes Lernen mit wissenschaftlicher Evidenz zu pädagogischer Diagnostik sollte zu mehr selbstgenerierten Informationen und damit einer positiven Einstellungsänderung resultieren. Entsprechend des ELM sollte darüber hinaus ein Gruppensetting das diagnostische Selbstkonzept besonders salient machen und zu einer positiven Einstellungsänderung führen.

Dementsprechend leiten folgende Forschungsfragen die Untersuchung an: Wie stark ist der Zusammenhang zwischen dem Diagnosewissen, der Einstellung zu Inklusion und dem diagnostischen Selbstkonzept bei Lehramtsstudierenden? Wie verändert sich dieser Zusammenhang im Verlauf eines Semesters? Zur Untersuchung dieser Fragestellungen werden die folgenden Hypothesen auf Grundlage einer Studie zum Training diagnostischer Kompetenz mit einer Form des selbstgesteuerten Lernens (problemorientiertes Lernen) an Textfällen (POL-TF) geprüft:

$>$ Hypothese a. Es besteht ein positiver Zusammenhang zwischen dem Diagnosewissen, der Einstellung zu Inklusion und dem diagnostischen Selbstkonzept.

$>$ Hypothese b. Die Zusammenhänge sind größer bei Personen, die selbstreguliert lernen, als bei Personen, die instruktionsbasiert lernen.

> Hypothese c. Die Zusammenhänge wachsen bei Personen, die selbstreguliert lernen, stärker als bei Personen, die instruktionsbasiert lernen. 


\section{Methoden}

\subsection{Stichprobe und Studiendesign}

Die vorliegende Studie hat ein quasi-experimentelles Design mit zwei Messzeitpunkten. An der Studie nahmen $N_{t 1}=23, N_{t 2}=18$ Lehramtsstudierende der beruflichen Fachrichtungen (Interventionsgruppe) und $N_{t 1}=40, N_{t 2}=26$ Lehramtsstudierende der Arbeitslehre (Kontrollgruppe) teil, die in jeweils einem von zwei parallel durchgeführten Seminaren zum Thema Diagnostik von Lernvoraussetzungen und -störungen im Wintersemester 2016/2017 teilnahmen. Die Teilnehmenden in der Interventionsgruppe waren 23-44 Jahre alt $(M=29,5$ Jahre, $S D=5,1 \mathrm{Jahre}, 78 \%$ weiblich), die Teilnehmenden in der Kontrollgruppe 21-39 Jahre $(M=26,4$ Jahre, $S D=4,6$ Jahre, $67 \%$ weiblich). Die Interventions- und Kontrollgruppe unterschieden sich weder signifikant in der Geschlechterverteilung $\left(\chi^{2}=2,017, p=0,365\right)$, noch im Alter $(U=525,500, z=-0,599, p=0,549)$.

Interventionsgruppe. Nach fünf ersten Lerneinheiten zu Grundlagen pädagogischer Diagnostik wurde den Studierenden POL vorgestellt. POL ist eine Unterrichtsmethode, die realitätsnahe Problemsituationen für selbstgesteuertes Lernen in Kleingruppen aufbereitet (Barrows 1985). Ein zentrales Merkmal von POL ist die kooperative Analyse eines Falls in einer festgelegten Schrittfolge. Dazu gehört das Lesen des Falls, die Diskussion fallbezogener Informationen und eigener themenbezogener Wissensbestände sowie die Entscheidung für weiterführende Lernziele, zum Beispiel die Symptome von Dyskalkulie erklären können. Anschließend bearbeiteten alle Gruppenmitglieder in Einzelarbeit alle Lernziele in Auseinandersetzung mit wissenschaftlicher Literatur, um ihre jeweiligen Lernergebnisse anschließend in der Kleingruppe zu diskutieren, zu dokumentieren und zu reflektieren. Zu Beginn der Intervention erhielten die Studierenden zusätzlich zum Fall Strukturierungshilfen (z.B. ein Arbeitsblatt zu POL-TF Schritten), anschließend wiederkehrende Übungsaufgaben (z. B. Hypothesenformulierung) und unterstützende Informationen in Form von Hinweisen zu Literatur sowie Literatur über Lernvoraussetzungen und -störungen. Die sechs verwendeten und von Expertinnen und Experten validierten Textfälle enthalten Informationen zu Schülerinnen und Schülern, zum Beispiel einleitende Narrationen, Notenlisten, Tests und ausgefüllte Beobachtungsbögen. Ausgewählt wurden Lernstörungen mit den höchsten Prävalenzraten unter Schülerinnen und Schülern, zum Beispiel Prüfungsangst oder Lese-Rechtschreibschwäche. Eine genauere Darstellung der Intervention findet sich an anderer Stelle (Wedel et al. 2019).

Kontrollgruppe. In der Kontrollgruppe besuchten die Studierenden ein thematisch identisches Seminar mit theoretischen Inputs und instruierter Kleingruppen- und Einzelarbeit. In jeweils einer Seminarstunde gab ein theoretischer Input durch den Do- 
zenten einen Überblick über die jeweilige Lernvoraussetzung oder -störung, wie etwa zu Ursache und Symptomen von Dyskalkulie sowie zu evidenzbasierten Interventionen. Im Anschluss wurde das verfügbare Wissen kritisch reflektiert, beispielsweise in methodischer und praktischer Hinsicht. In der verbleibenden Zeit leitete der Dozent oder eine Gruppe der Teilnehmenden eine Übung zur Anwendung des erworbenen Wissens an, zum Beispiel ein Gruppenpuzzle zu aufmerksamkeitsförderlicher Klassenraumgestaltung. Den Teilnehmenden wurde dieselbe Literatur wie der Interventionsgruppe zur Verfügung gestellt. Die Kontroll- und Interventionsgruppe wurden durch denselben Dozenten angeleitet und ihnen wurden exakt dieselben Lernziele sowohl für das gesamte Seminar als auch die jeweiligen Themen mitgeteilt.

\subsection{Messinstrumente}

Folgende Konstrukte wurden mit einem papierbasiertem Fragebogen in der berichteten Reihenfolge gemessen.

Die Einstellung zu inklusivem Unterrichten wurden mit der Selbsteinschätzungsskala EFI-L von Seyfried und Heyl (2016) gemessen, wobei lediglich die Subskala zur Bereitschaft für inklusiven Unterricht ausgewählt wurde, da die Studierenden noch nicht im Lehrberuf eingemündet waren. Die verwendete Subskala EFI-L erfasst die Einstellung mit fünf Items auf einer sechsstufigen Antwortskala von $1=$ stimme ganz und gar nicht $z u$ bis $6=$ stimme voll und ganz $z u$. Enthalten sind Items zur affektiven Einstellungskomponente, zum Beispiel »Der Unterricht in einer inklusiven Klasse macht mir Angst.« (rekodiert) sowie zur kognitiven Einstellungskomponente, zum Beispiel »Ich kann mir vorstellen, im kommenden Schuljahr in einer inklusiven Klasse zu unterrichten. «Die Reliabilität betrug für die Interventionsgruppe $\alpha_{\mathrm{t} 1 / \mathrm{t} 2}=0,75 / 0,62$ und für die Kontrollgruppe $\alpha_{\mathrm{t} 1 / \mathrm{t} 2}=0,68 / 0,75$.

Das Selbstkonzept wurde mit der Lehrkräfte-Selbsteinschätzungsskala zum diagnostischen Selbstkonzept (LSD) von Wedel und Müller (2017) gemessen. 15 Items erfassen auf einer Antwortskala von $1=$ trifft gar nicht $z u$ bis $4=$ trifft genau $z u$, wie die Studierenden ihre Fähigkeiten in pädagogischer Diagnostik einschätzen, zum Beispiel »Ich kann Instrumente zur Erfassung von Lernvoraussetzungen einsetzen (z. B. Beobachtungsbögen, Tests).« Die Reliabilität der Skala betrug für die Interventionsgruppe $\alpha_{\mathrm{t} 1 / \mathrm{t} 2}=0,94 / 0,91$ und für die Kontrollgruppe $\alpha_{\mathrm{t} 1 / \mathrm{t} 2}=0,91 / 0,91$.

Das Wissen zu pädagogischer Diagnostik wurde mit einem objektiven Wissenstest zum pädagogischen Diagnosewissen (PDW) von Wedel und Müller (2016) erfasst. Der PDW erfasst kognitive Fähigkeiten im Erkennen, Verstehen und Anwenden in den fünf aufeinander folgenden Inhaltsbereichen: Urteilsfehler, Feedback, Ablauf pädagogischer Diagnostik, individuelle Lernvoraussetzungen und Testgütekriterien. In der 
vorliegenden Studie werden die kognitiven Fähigkeiten im Verstehen analysiert, da diese den Kriterien hoher kognitiver Elaboration im ELM entsprechen. Das Verstehen der zentralen Inhaltbereiche des Diagnosewissens wurden mit zehn Multiple-Choice-Items (zwei Items je Inhaltsbereich) erfasst, zum Beispiel »Sie korrigieren einen schriftlichen Test Ihrer Klasse. Die ersten zehn Tests fallen durchgehend schlecht aus. Worauf achten Sie bei der weiteren Korrektur besonders? «. Jedes Item wurde entweder als richtig oder falsch codiert, sodass im Bereich des Verstehens maximal zehn Punkte erreicht werden konnten (Range 0-10). Da der Test in zwei parallelen Versionen vorliegt, konnten Testwiederholungseffekte ausgeschlossen werden.

\subsection{Analyseverfahren und messwiederholte Korrelation}

Die Hypothesen wurden mit Korrelationsanalysen und Unterschiedstests ( $t$-Tests für Korrelations- und Regressionskoeffizienten) geprüft. Eine methodische Herausforderung liegt dabei in der Kombination aus Zusammenhangsanalyse, der vorliegenden Datenstruktur und der kleinen Stichprobe.

Zusammenhangsanalysen (z. B. Korrelationen und Regressionen) basieren auf der Annahme unabhängiger Residuen bzw. Messfehler (Eid, Gollwitzer und Schmidt 2011). Mit anderen Worten dürfen zwar die gemessenen Konstrukte miteinander korrelieren, nicht aber die individuellen Abweichungen vom Erwartungswert, die bei der Datenerhebung entstehen. Diese Annahme kann in bestimmten Situationen allerdings nicht getroffen werden, beispielsweise wenn die Datenerhebung für einen Teil der Stichprobe systematisch durch Störgrößen beeinflusst wird oder wenn von ein- und derselben Person mehrfach Messwerte erhoben werden. Die paarweise Abhängigkeit durch Messwiederholung ist dabei der häufigste Fall und trifft auch für die vorliegende Studie zu.

Für größere Stichproben kann diese Autokorrelation als eigener Term in Modelle integriert und die eigentliche Zusammenhangsmessung davon bereinigt werden, zum Beispiel in Multi-Level-Modellen, latenten Wachstumskurvenmodellen oder Autoregressiven Distributed Lag Modellen (ARDL). Da diese Modelle für kleine Stichproben nicht identifizierbar sind, braucht es andere Strategien, um mit einer Verletzung der Unabhängigkeit von Residuen umzugehen.

Nicht in der Standardliteratur behandelt ist das speziell für Einschränkungen in der Unabhängigkeit der Residuen entwickelte messwiederholte Korrelationsmaß $r_{r m}$ (Bakdash und Marusich 2017; Bland und Altmann 1995). Da die Darstellung von $r_{r m}$ in Bland und Altmann (1995) nicht ausformuliert ist und in Bakdash und Marusich (2017) nachkorrigiert wurde, erfolgt an dieser Stelle die vollständige Fassung (siehe auch: Bakdash und Marusich 2019). 
Die messwiederholte Korrelation $r_{r m}$ modelliert den Zusammenhang von Konstrukten als einfaktorielle Kovarianzanalyse (ANCOVA). Während in der Varianzanalyse üblicherweise Unterschiede zwischen Personengruppen getestet werden, sieht $r_{r m}$ vor, dass jede Person eine $>$ Gruppe $<$ bzw. einen Faktor $(j)$ bildet und die paarweise abhängigen Messwerte einer Person eines Konstrukts die > Gruppenmitglieder< bzw. Faktorstufen (i). Das zweite Konstrukt ist die Kovariate. Um einen für alle Personen geltenden Zusammenhang zu schätzen, wird eine, für alle Messwiederholungspaare am besten passende Beziehung zwischen den zwei korrelierenden Konstrukten geschätzt, das heißt eine Schar von Regressionsgeraden mit gleichem Anstieg, aber unterschiedlichem Schnittpunkt mit der y-Achse. Mit anderen Worten wird die Varianz zwischen den Personen genutzt, um den durch die autokorrelative Datenstruktur veränderten Zusammenhang zu korrigieren.

Es sei:

$$
Y_{i j}=\mu+\tau_{j}+\beta\left(X_{i j}-\bar{X}\right)+\varepsilon_{i j}
$$

mit

$Y_{i j} \ldots$ der Messwert der abhängigen bzw. ersten Variable für den $i$-ten Messwert im $j$-ten Faktor, mit $j=1$...p, $i=1 \ldots \mathrm{q}$

$\mu$... der Gesamtmittelwert $\overline{Y_{\imath J}}$

$\tau_{j} \ldots$ der Effekt des $j$ Faktors (Person)

$\beta$... der Anstieg der Kovariate bzw. der zweiten Variable

$X_{i j} \ldots$ der Messwert der Kovariate für den i-ten Messwert im $j$-ten Faktor

$\bar{X}$... der Mittelwert aller Werte $X_{i j}$

$\varepsilon_{i j} \ldots$ der Messfehler

berechnet durch

$\mu=\frac{\sum_{i=1}^{\mathrm{q}} \sum_{j=1}^{\mathrm{p}}\left(Y_{i j}\right)}{\mathrm{qp}}$, mit $q p \ldots$ Anzahl der gemessenen Werte in $Y_{i j}$

$\tau_{j}=\sum_{j=1}^{\mathrm{p}}\left(\overline{Y_{\cdot j}}-\mu\right)$, mit $\overline{Y_{\cdot j}} \ldots$ der Mittelwert des $j$-ten Faktors

$\beta=$ Schätzung durch Ordinary Least Squares (OLS) für alle Messwertpaare

$\varepsilon_{i j}=\sum_{i=1}^{\mathrm{q}} \sum_{j=1}^{\mathrm{p}}\left(Y_{i j}-\overline{Y_{\cdot \mathrm{j}}}\right)$ 
Der Korrelationskoeffizient $r_{r m}$ wird anschließend durch die Varianzanteile der Kovariate und der Residuen berechnet.

$$
r_{r m}=\sqrt{\frac{\text { SAQ der Kovariate }}{\text { SAQ der Kovariate }+ \text { SAQ der Residuen }}}
$$

mit

SAQ ... Summe der Abweichungsquadrate bzw. sum of squares' (SS)

berechnet durch

klassische Schritte der Kovarianzanalyse (Rasch und Schott 2018, 495), die auch in statistischer Standardsoftware implementiert sind.

Das Vorzeichen des Anstiegs $\beta$ der Kovariate bestimmt dabei das Vorzeichen des Korrelationskoeffizienten. Der Parameter $r_{r m}$ bewegt sich zwischen -1 und +1 und ist analog zu Pearson's $r$ zu interpretieren. Bei -1 liegt ein perfekter negativer Zusammenhang zwischen zwei Konstrukten vor, bei +1 ein perfekter positiver Zusammenhang und bei 0 kein Zusammenhang. Darüber hinaus zeigt der Anstieg $\beta$ auch die Veränderung des Zusammenhangs für die jeweiligen Personen an.

\subsection{Berechnungsschritte für $r_{r m}$ in Statistiksoftware}

Die Berechnung von $r_{r m}$ kann sowohl in SPSS als auch in R erfolgen (hier dargestellt mit SPSS 25 und R 3.5.1). Für beide Programme müssen die Daten im Langformat vorliegen, das heißt, die Messwertpaare der Konstrukte bekommen für jede Messwiederholung eine eigene Datenzeile, die um die personenidentifizierende Variable, zum Beispiel ein anonymisierter Code, ergänzt wird. Insgesamt sollten dementsprechend drei Datenspalten vorliegen (personenidentifizierende Variable, erste Variable [aV], zweite Variable [Kovariate]). Für jede Person entspricht die Anzahl der Datenzeilen dann der Anzahl der Messwiederholungen, wobei die personenidentifizierende Variable in jeder Datenzeile wiederholt wird.

In SPSS:

1. Analysieren $>$ Allgemeines Lineares Modell $>$ Univariat

2. Erste Variable als abhängige Variable eingeben, zweite Variable als Kovariate und personenidentifizierende Variable als festen Faktor. OK klicken.

3. Im Output der Tabelle »Test auf Zwischensubjekteffekte « in der Spalte »Quadratsumme vom Typ III « die Werte der Kovariate und Fehler entnehmen.

4. In Gleichung 2 einsetzen und Vorzeichen anpassen. 
In R:

1. Paket rmcorr (Bakdash und Marusich 2017) laden und Datensatz einlesen

2. Funktion rmcorr aufrufen (siehe Hilfedatei des Pakets) und Datensatz sowie Variablennamen einfügen. Ausführen.

\subsection{Ergebnisse}

Deskriptiv gaben die Lehramtsstudierenden eine eher positive Einstellung zu Inklusion an und hatten mittlere bis eher geringe kognitive Fähigkeiten in Bereich Verstehen des Diagnosewissens (siehe Tabelle 1).

\begin{tabular}{lcccccc} 
& \multicolumn{2}{c}{ Alle Teilnehmenden } & \multicolumn{2}{c}{ Interventionsgruppe } & \multicolumn{2}{c}{ Kontrollgruppe } \\
& $\mathbf{t 1}$ & $\mathbf{t 2}$ & $\mathbf{t 1}$ & $\mathbf{t 2}$ & $\mathbf{t 1}$ & $\mathbf{t 2}$ \\
& $\mathbf{n = 6 3}$ & $\mathbf{n = 4 4}$ & $\mathbf{n = 2 3}$ & $\mathbf{n = 1 8}$ & $\mathbf{n = 4 0}$ & $\mathbf{n = 2 6}$ \\
EFI-L & 3.35 & 3.38 & 3.69 & 3.73 & 3.15 & 3.14 \\
& $(0.91)$ & $(0.77)$ & $(0.99)$ & $(0.67)$ & $(0.81)$ & $(0.75)$ \\
PDW-V & 3.86 & 4.28 & 4.04 & 5.44 & 3.75 & 3.44 \\
& $(1.52)$ & $(1.83)$ & $(1.52)$ & $(1.15)$ & $(1.53)$ & $(1.78)$ \\
LSD & 2.59 & 2.87 & 2.49 & 2.92 & 2.65 & 2.83 \\
& $(0.59)$ & $(0.42)$ & $(0.62)$ & $(0.43)$ & $(0.57)$ & $(0.42)$ \\
\hline
\end{tabular}

Tabelle 1: Mittelwerte und Standardabweichungen (in Klammern) für alle Teilnehmenden und Interventions- und Kontrollgruppe (Anmerkungen: $\mathrm{t} 1$ = Erster Messzeitpunkt, t2 = Zweiter Messzeitpunkt, EFI-L = Einstellungen zur Bereitschaft inklusiven Unterrichtens Skala 1-6, PDW-V = Wissen zu pädagogischer Diagnostik Verstehen Skala 1-10, LSD = Lehrkräfte Selbsteinschätzung zum diagnostischen Selbstkonzept Skala $1-4)$

Mann-Whitney-U Tests ergaben, dass sich die Interventions- und Kontrollgruppe zu $t_{1}$ in den Einstellungen zu inklusivem Unterrichten unterschieden (EFI-L, $z=2,084$, $p=0,037$ ), nicht aber im Diagnosewissen (PDW-V, $z=0,497, p=0,497$ ) oder im diagnostischen Selbstkonzept (LSD, $z=-0,858$, $p=0,391$ ). Zu $t_{2}$ unterschieden sich die Gruppen in Bezug auf EFI-L $(z=2,463, p=0,014)$ und auf das PDW-V $(z=3,718$, $p<0,001)$, aber nicht in Bezug auf $\operatorname{LSD}(z=0,778, p=0,737)$. 


\subsection{Zusammenhangsanalysen}

Hypothese a betraf die Annahme eines positiven Zusammenhangs zwischen Einstellungen zu inklusivem Unterrichten, Diagnosewissen und dem diagnostischen Selbstkonzept. Die Prüfung erfolgte mit einer messwiederholten Korrelation $r_{r m}$ in der Interventions- und Kontrollgruppe.

Die zum Teil beträchtlichen Autokorrelationen zwischen den zwei Messzeitpunkten betrugen in der Kontrollgruppe EFI-L $r=0,455, p=0,044$; PDW-V $r=0,270, p=0,236$; LSD $r=0,209, p=0,376$ und in der Interventionsgruppe EFI-L $r=0,288, p=0,318$; PDW-V $r=-0,006, p=0,982$; LSD $r=0,552, p=0,041$. Dies ist eine typische Eigenschaft von reflektiven Konstrukten, die ohne Berücksichtigung im Schätzverfahren zu größeren Ungenauigkeiten führt. Die folgende Tabelle stellt die messwiederholte Korrelation, die diese autokorrelative Struktur berücksichtigt, möglichen Pearson Korrelationen, die diese Datenstruktur nicht berücksichtigt, zur Veranschaulichung gegenüber (siehe Tabelle 2).

\begin{tabular}{|c|c|c|c|c|c|}
\hline & & \multicolumn{2}{|c|}{ Kontrollgruppe } & \multicolumn{2}{|c|}{ Interventionsgruppe } \\
\hline & & EFI-L & PDW-V & EFI-L & PDW-V \\
\hline \multirow[t]{4}{*}{ PDW-V } & rignore & $.167(.218)$ & & $.076(.658)$ & \\
\hline & $r_{\text {meanbetween }}$ & $.171(.312)$ & & $.171(.321)$ & \\
\hline & $r_{\text {mean within }}$ & $.170(.294)$ & & $.003(.989)$ & \\
\hline & $r_{m}$ & $.202(.436)$ & & $.262(.366)$ & \\
\hline \multirow[t]{4}{*}{ LSD } & rignore & $.391(.001)$ & $-.079(.565)$ & $.471(.002)$ & $.081(.641)$ \\
\hline & $r_{\text {meanbetween }}$ & $.398(.002)$ & $-.049(.812)$ & $.477(.029)$ & $-.099(.696)$ \\
\hline & $r_{\text {mean within }}$ & $.253(.115)$ & $-.166(.313)$ & $.563(.005)$ & $-.252(.259)$ \\
\hline & $r_{m}$ & $.667(.001)$ & $.313(.221)$ & $-.126(.652)$ & $.505(.065)$ \\
\hline
\end{tabular}

Tabelle 2: Korrelationen in Pearsons $r$ zwischen Einstellungen, Wissen und Selbstkonzept und zugehörige p-Werte (in Klammern) (Anmerkungen: EFI-L = Einstellungen zur Bereitschaft inklusiven Unterrichtens Skala 1-6, PDW-V = Wissen zu pädagogischer Diagnostik Verstehen Skala 1-10, LSD = Lehrkräfte Selbsteinschätzung zum diagnostischen Selbstkonzept Skala 1-4)

Dafür wurden folgende Pearson Korrelationen gebildet, die sich auch in der Forschungsliteratur zu Interventionen für Einstellungsänderungen im Bereich schulischer Inklusion finden (z. B. Batsiou et al. 2008), jedoch zu fehlerhaften Ergebnissen führen können: Die Korrelation $\left(r_{\text {ignore }}\right)$ behandelt jeden Datenpunkt als ein vollständiges Element der Population, das heißt, es wird ignoriert, dass mehrere Datenpunkte von 

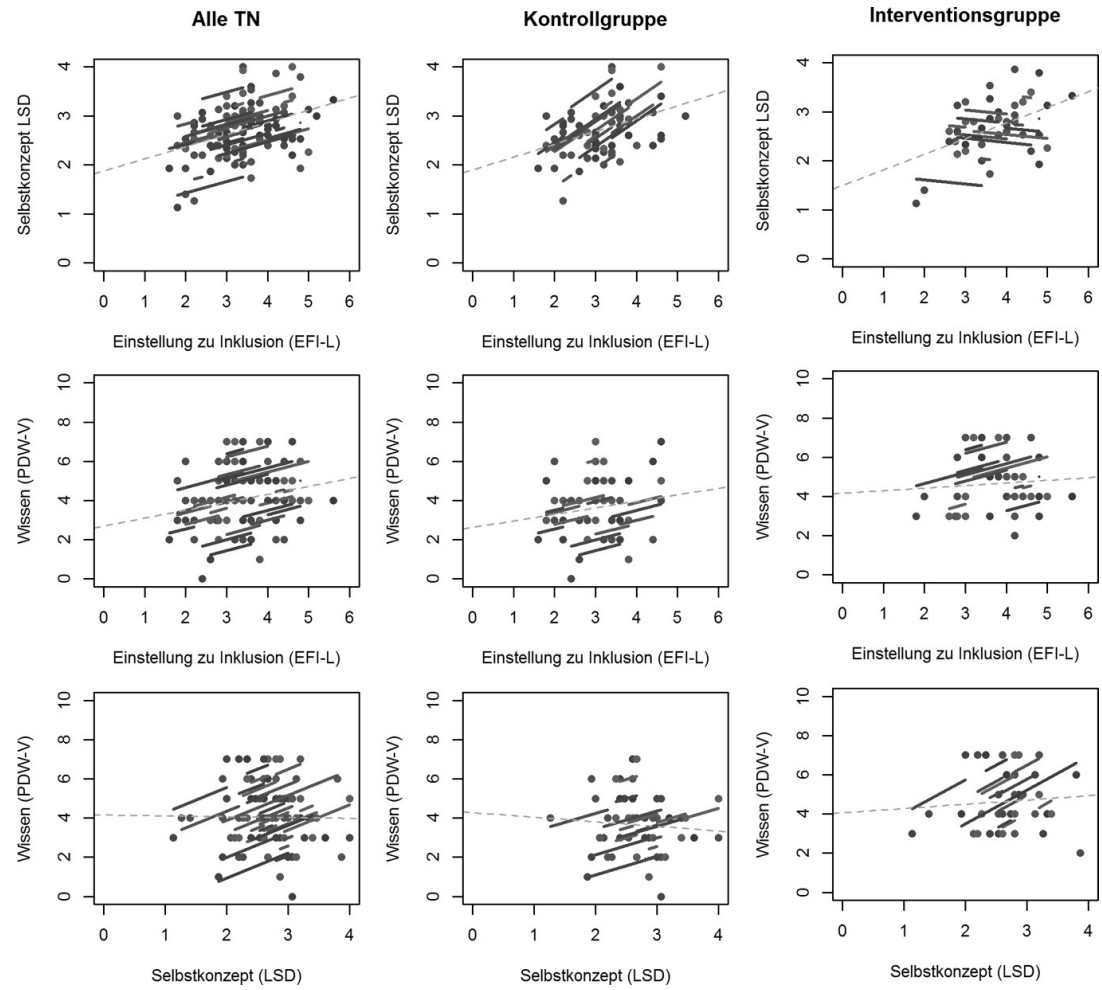

Abbildung 1: Streudiagramme für Einstellung zu Inklusion, Diagnosewissen und diagnostisches Selbstkonzept (Anmerkung: In den Streudiagrammen stelltjeder Punkt einen Messwert einer Person zu einem Messzeitpunkt dar. Die Messwertpaare, d. h. Personen, sind durch Graustufen unterschieden, Pearson-Korrelationen ohne Berücksichtigung der Dtaenstruktur sind mit einer gesrichelten Linie eingezeichnet. Die Funktionsschaar der messwiederholten Korrelation ist mit durchgezogenen Linien eingezeichnet.)

derselben Person stammen und daher autokorreliert sind. Die Korrelation $\left(r_{\text {mean_within }}\right)$ behandelt die Mittelwerte der messwiederholten Datenpunkte jeder Person als vollständiges Element der Population, das heißt, es werden für jede Person für jedes Konstrukt zuerst ein Mittelwert aller für diese Person verfügbaren Datenpunkte gebildet und anschließend diese Mittelwerte korreliert. Für die Korrelation $\left(r_{\text {mean_between }}\right)$ werden zuerst Korrelationen der Konstrukte innerhalb jedes Messzeitpunktes berechnet und dann deren Mittelwert gebildet.

Eine besonders deutliche Abweichung besteht zwischen $r_{\text {ignore }}$ und $r_{\mathrm{rm}}$. Trägt man beide grafisch in ein Streudiagram ein (siehe Abbildung 1), fällt insbesondere der Zusam- 
menhang zwischen dem diagnostischen Selbstkonzept und der Einstellung zu Inklusion in der Interventionsgruppe sowie der Zusammenhang zwischen dem Diagnosewissen und dem diagnostischen Selbstkonzept in der Kontrollgruppe auf. Beide Zusammenhänge zeigen ein Simpson Paradoxon (Kievit, Frankenhuis, Waldorp und Borsboom 2013), das heißt, auf Populations- und auf Individualebene bestehen gegenläufige Zusammenhänge. In der Interventionsgruppe zeigte sich etwa, dass auf Ebene der Gesamtgruppe ein positiver Zusammenhang zwischen Einstellung und Selbstkonzept besteht. Korrekt auf Individualebene gemessen war dieser Zusammenhang jedoch negativ (siehe Abbildung 1).

In der Kontrollgruppe gab es einen mittleren, aber nicht signifikanten positiven Zusammenhang zwischen dem Diagnosewissen und den Einstellungen zu inklusivem Unterrichten $\left(r_{r m}=0,202, p=0,436\right)$. Dagegen gab es einen großen, signifikanten positiven Zusammenhang der Einstellungen zu inklusivem Unterrichten mit dem diagnostischen Selbstkonzept $\left(r_{r m}=0,667, p=0,001\right)$. Diagnosewissen und diagnostisches Selbstkonzept hatten einen mittleren positiven, aber nicht signifikanten Zusammenhang $\left(r_{r m}=0,311, p=0,221\right)$.

In der Interventionsgruppe war der Zusammenhang zwischen dem Diagnosewissen und den Einstellungen zu inklusivem Unterrichten positiv und von mittlerer Größe, aber nicht signifikant $\left(r_{r m}=0,262, p=0,366\right)$. Ein negativer, aber nicht signifikanter Zusammenhang bestand zwischen der Einstellung zu Inklusion und dem diagnostischen Selbstkonzept $\left(r_{r m}=-0,126, p=0,652\right)$. Dagegen zeigte sich ein großer und marginal signifikanter positiver Zusammenhang zwischen dem diagnostischen Selbstkonzept und dem Diagnosewissen $\left(r_{r m}=0,505, p=0,065\right)$.

Insgesamt kann Hypothese a trotz der positiven mittleren Effektgrößen nur für den Zusammenhang von Einstellungen zu inklusivem Unterrichten und dem diagnostischen Selbstkonzept für die Kontrollgruppe und für den Zusammenhang von Diagnosewissen und diagnostischem Selbstkonzept in der Interventionsgruppe bestätigt werden.

\subsection{Unterschiedstests und Zusammenhangsveränderungen}

Hypothese b trifft die Aussage, dass die Zusammenhänge in der Interventionsgruppe größer ausfallen als in der Kontrollgruppe. Zur Prüfung wurden $z$-Tests für Korrelationsunterschiede zwischen Kontroll- und Interventionsgruppe berechnet (Eid, Gollwitzer und Schmidt 2011, 547).

Im Korrelationsmaß $r_{r m}$ bestand kein Unterschied zwischen den Gruppen für den Zusammenhang von PDW-V und EFI-L $(z=-0,229, p=0,410)$, aber ein signifikanter Unterschied für den Zusammenhang von LSD und EFI-L $(z=-3,358, p<0,001)$. Für den Zusammenhang von PDW-V und LSD bestand kein Unterschied zwischen den Gruppen $(z=-0,836, p=0,201)$. 
Insgesamt kann Hypothese b daher nur für den Zusammenhang von Einstellung zu Inklusion mit dem diagnostischen Selbstkonzept beibehalten werden.

Hypothese $\mathrm{c}$ trifft die Aussage, dass die Zusammenhänge in Interventionsgruppe stärker wachsen als in der Kontrollgruppe. Zur Prüfung wurden Unterschiede zwischen der Interventions- und Kontrollgruppe in der Veränderung der Korrelationsmaße durch $t$-Tests für den Anstieg der Kovariate, das heißt, für den $\beta$-Koeffizienten berechnet (Cohen et al. 2003). Damit können Aussagen über Unterschiede in den Veränderungen von Zusammenhängen getroffen werden, was üblicherweise nur mit latenten Wachstumskurvenmodellen und entsprechend umfangreichen Stichproben möglich ist. In Simulationsstudien liegt die für eine ausreichende Teststärke benötigte Stichprobengröße für Wachstumskurvenmodelle bei 200 bis 500 Personen mit vier bis sechs Messzeitpunkten (Hamilton und Hancock 2003) und für ANCOVAs bei 30 bis 60 Personen (Seaman, Algina und Oljenik 1985). Trotz dieses großen Vorteils hat das Verfahren des $\beta$-Vergleichs aber auch typische Einschränkungen manifester Modellspezifikation, beispielsweise werden Messfehler und Methodenfaktoren nicht explizit geschätzt.

Zwischen den Gruppen bestand ein Unterschied in der Veränderung im Zusammenhang von EFI-L und LSD $(t(67)=2,104, p=0,039)$. In der Interventionsgruppe sank dieser Zusammenhang im Verlauf des Semesters ab $(\beta=-0,08)$, während er in der Kontrollgruppe anstieg $(\beta=0,57)$ (siehe Abbildung 1 ). Für die Veränderung des Zusammenhangs von PDW-V und EFI-L sowie von PDW-V und LSD bestand dagegen kein Unterschied zwischen den Gruppen $\left(\beta_{\text {Interventionsgruppe }}=0,57, \beta_{\text {Kontrollgruppe }}=0,53\right.$, $t(57)=0,046, p=0,964 ; \beta_{\text {Interventionsgruppe }}=1,72, \beta_{\text {Kontrollgruppe }}=0,93, t(57)=0,398$, $p=0,692)$.

Hypothese c kann daher nur für den Zusammenhang von Einstellung zu inklusivem Unterrichten und diagnostischem Selbstkonzept beibehalten werden.

\section{Diskussion}

\subsection{Zusammenhänge von Diagnosewissen, Einstellung zu inklusivem Unterrichten und diagnostischem Selbstkonzept}

\section{Diagnosewissen und Einstellung zu inklusivem Unterrichten}

In beiden Untersuchungsgruppen konnte ein mittlerer positiver, aber nicht signifikanter Zusammenhang festgestellt werden. Die Richtung dieses Zusammenhangs entspricht den Modellen professioneller Lehrkompetenz. Dabei bestand weder ein Gruppenunterschied in der Stärke des Zusammenhangs noch in dessen Anstieg im Semesterverlauf. Die Ergebnisse entsprechen also nur eingeschränkt den Aussagen des ELM. Es kann zu- 
dem entgegen empirischen Ergebnissen des ELM zu selbstgesteuertem Lernen (Darke et al. 1998; Wenden 1998) nicht davon ausgegangen werden, dass POL eine wirksamere Methode zur stärkeren Verknüpfung von Diagnosewissen und Einstellungen zu inklusivem Unterrichten darstellt als instruktionsbasiertes Lernen. Eine Erklärung dafür liegt in der Verfügbarkeit starker Argumente in Form wissenschaftlicher Evidenz in beiden Untersuchungsgruppen. Möglicherweise überschattete dies den Effekt des selbstgesteuerten Lernens.

\section{Diagnostisches Selbstkonzept und Einstellung zu inklusivem Unterrichten}

Während in der Kontrollgruppe ein starker signifikant positiver Zusammenhang bestand, konnte in der Interventionsgruppe ein schwacher negativer Zusammenhang festgestellt werden. Zwischen den Gruppen war sowohl dieser Unterschied signifikant als auch dessen Veränderung. Dabei wuchs der Zusammenhang in der Kontrollgruppe, während er in der Interventionsgruppe schrumpfte. Besonders letzteres Ergebnis widerspricht der Aussage der > functional matching < Hypothese des ELM. Eine mögliche Erklärung dafür ist die Zusammenarbeit in den POL-Kleingruppen. Die Lehramtsstudierenden waren mit der Methode nicht vertraut und zeigten anfänglich Schwierigkeiten in der Festlegung gemeinsamer Lernziele und der Strukturierung von Diskussionen. Wie Brownell und andere (2006) anmerken, braucht gelingende Kooperation zwischen Lehrkräften kontextspezifische Kommunikationsfähigkeiten, da sich pädagogische und diagnostische Handlungs- und Entscheidungsstrategien zwischen angehenden Lehrkräften unterscheiden können. Es ist plausibel anzunehmen, dass Diskussionen über Maßnahmen im Bereich schulischer Inklusion Konfliktpotenzial in sich tragen, was die wahrgenommene Gruppenzugehörigkeit verringern kann.

\section{Diagnostisches Selbstkonzept und Diagnosewissen}

In beiden Gruppen bestand ein theoriekonformer mittlerer bis großer positiver Zusammenhang, der jedoch nur in der Interventionsgruppe signifikant wurde. Dabei bestand kein Unterschied zwischen den Gruppen und ebenfalls kein Unterschied bzgl. der Zusammenhangsveränderung. Auch hier kann also nicht von einem differenziellen Effekt von POL ausgegangen werden.

\subsection{Evidenz zu Diagnosewissen und dem Selbstkonzept als Basis der Einstellung zu inklusivem Unterrichten}

Die evidenzbasierte Konstruktion von Lerngelegenheiten zu inklusivem Unterrichten stellt ein wichtiges Lernfeld für die Lehrkräftebildung dar. Die vorliegende Interventionsstudie trägt zwei zentrale Ergebnisse zu dieser Diskussion bei. 
Die bisherigen Forschungsbefunde zum querschnittlichen Zusammenhang von Diagnosewissen und Einstellung zu inklusivem Unterrichten entsprachen auf deskriptiver Ebene den Ergebnissen der vorliegenden Studie. Während in bisherigen Studien oft auf Selbsteinschätzungen zum Wissen zurückgegriffen wurde (z. B. Sermier Dessemontet, Benoit und Bless 2011), zeigt die vorliegende Studie ähnliche deskriptive Zusammenhänge auch mit einem objektiven Wissenstest und einer stringenteren statistischen Analyse. Darüber hinaus konnte auf deskriptiver Ebene ein wachsender Zusammenhang dieser Konstrukte im Lernverlauf festgestellt werden. Dieser Befund unterstützt die konzeptuelle Annahme der Modelle professioneller Lehrkompetenz, dass Diagnosewissen eine Grundlage der Einstellung zu inklusivem Unterrichten bildet. Dieses Ergebnis bestand unabhängig von dem Format, in dem Lehramtsstudierende Wissen zu pädagogischer Diagnostik erwarben.

Demgegenüber waren der Zusammenhang von Selbstkonzept und Einstellungen zu inklusivem Unterrichten sowie dessen Veränderung vom Lernformat abhängig. Der schrumpfende Zusammenhang in der Gruppe mit selbstgesteuertem Lernen zeigt exemplarisch, dass Lerngelegenheiten mit dem Ziel der Vermittlung von Diagnosewissen nicht in jedem Fall zu einer Verknüpfung von diagnostischem Selbstkonzept und Einstellungen zu inklusivem Unterrichten führen. Möglicherweise braucht es dazu besondere Instruktions- oder Reflexionsformen, die in den untersuchten Lerngelegenheiten nicht angeboten wurden. Ein Hinweis darauf ist etwa, dass eigene Vorerfahrungen mit inklusiven Kontexten zu den stärksten Einstellungsprädiktoren gehören (de Boer, Pijl und Minnaert 2011). Zu diesen Erfahrungen und ihrer Reflexion bieten etwa die Praxisanteile der Lehramtsausbildung ein großes Potenzial.

Studien zum Übergang angehender Lehrkräfte in die schulpraktische Arbeitswelt zeigen aber auch, dass sich häufig eine starke Orientierung an vorgefundenen Unterrichtsskripts, pädagogischen Praktiken und schulinternen Normen einstellt (Hargreaves 2000; Santagata und Yeh 2016). Diese Studien stellen für das Thema der Evidenzbasierung infrage, welche Wirksamkeitskriterien in der Entwicklung von Lerngelegenheiten angelegt werden. Im Hinblick auf diese Instabilität erworbener Kompetenzen besteht dringender Bedarf an wissenschaftlicher Evidenz, die über Angebots-Nutzen-Modelle für den Zeitraum der Kompetenzvermittlung in der ersten Phase der Lehrkräftebildung hinausgeht.

\subsection{Messwiederholte Korrelation und manifeste Wachstumskurvenmodelle}

Die Berechnung von Zusammenhangsveränderung, das heißt des Unterschieds oder Zusammenhangs von Zusammenhängen über die Zeit oder zwischen Gruppen stellt 
mit klassischen probabilistischen Modellen hohe Anforderungen an die Stichprobe und mit bayesianischen Modellen hohe Anforderungen an methodische Kenntnisse. Die messwiederholte Korrelation und das manifeste Wachstumskurvenmodell bieten einen Kompromiss an, der wünschenswerte Eigenschaften beider Modelle vereint und für die Anwendung in der Lehrkräftebildungsforschung hinnehmbare Einschränkungen aufweist. Die Berechnung und Prüfung des Wachsens oder Schrumpfens von Zusammenhängen in kleinen Stichproben eröffnet der Lehrkräftebildung die Untersuchung von Fragestellungen, die unter den strukturellen Bedingungen des Bildungssystems für angehende Lehrkräfte anderweitig nicht an jedem Standort der Lehrkräftebildung möglich wäre. Da bisher nur wenige Untersuchungen im Bildungsbereich mit dieser Messgröße vorliegen, können Einschätzungen zur Effektgröße nur durch den Vergleich mit verwandten Korrelationsmaßen getroffen werden. Da die messwiederholte Korrelation in anderen Wissenschaftsbereichen (z. B. Medizin, Biologie) bereits zunehmend genutzt wird, ist dies jedoch auch in der empirischen Bildungsforschung absehbar.

\subsection{Grenzen der Studie}

Die Zusammenhangsanalysen betreffen einen sehr spezifischen Kontext, aus dem nicht ohne weiteres auf andere Lerngelegenheiten in der Lehrkräftebildung geschlossen werden kann. Wünschenswert wären weitere Stichproben, in denen Diagnosewissen mit anderen didaktischen Methoden vermittelt wird oder aber mit derselben Methode in einem anderen institutionellen Kontext. Es ist nicht auszuschließen, dass die ermittelten Zusammenhänge charakteristisch für die spezifische Stichprobe sind.

Die geringe Stichprobengröße bedeutet für viele der gezeigten Zusammenhänge eine Unsicherheit in Bezug auf ihre Signifikanz. Die berechneten Zusammenhänge fallen deskriptiv durchgängig in die theoretisch zu erwartende Richtung aus, sie können jedoch nicht mit hinreichender Sicherheit angenommen werden. Da die Effektgrößen jedoch zum Teil als sehr groß eingestuft werden können, sollte der Signifikanzwert nicht als einziges Kriterium in die Interpretation einbezogen werden.

Darüber hinaus konnten die Teilnehmenden aus studienorganisatorischen Gründen nicht randomisiert zur Interventions- und Kontrollgruppe zugeordnet werden. Diese Limitation wirkt jedoch weniger gewichtig, da die Gruppen in den gemessenen Konstrukten zum ersten Messzeitpunkt eine gute Vergleichbarkeit zeigten.

Dass die Einstellungsskala nur akzeptable Reliabilitäten aufwies, ist möglicherweise auf die kleine Stichprobengröße im Verhältnis zur Anzahl der Items zurückzuführen. Da die Skala umfangreich validiert wurde, kann jedoch von ihrer prinzipiellen Zuverlässigkeit ausgegangen werden. Darüber hinaus wurde die Einstellung zu inklusivem Unterrichten vor dem Wissenstest abgefragt, um eine Konfundierung auszuschließen. 
Mit dem diagnostischen Selbstkonzept wurde nur eine Facette des professionellen Selbstkonzepts von Lehramtsstudierenden und Lehrkräften gemessen, die jedoch konzeptionell in einem wichtigen Zusammenhang mit dem Diagnosewissen steht. Vor dem Hintergrund der bisherigen Forschung, in der überwiegend nur eines dieser Konstrukte gemessen wird, stellt dies jedoch einen Erkenntnisgewinn dar.

Da keine weiteren Messzeitpunkte vorliegen, bleibt auch offen, inwiefern sich der Zusammenhang zwischen Diagnosewissen und Einstellung zu inklusivem Unterrichten durch Praxiserfahrungen im Schulkontext verändert. Denkbar wäre sowohl ein Anstieg des Zusammenhangs in Anlehnung an die Kontakthypothese (Allport 1954), als auch ein Abfall durch den sogenannten >Praxisschock $<$.

\subsection{Ausblick}

Die Förderung inklusionsbejahender Einstellungen ist eine zentrale Voraussetzung für lernwirksames Unterrichten in heterogenen Klassen. Aktuelle Modelle professioneller Lehrkompetenzen konzipieren diagnostische Kompetenz als eine Voraussetzung für erfolgreiches Unterrichtshandeln in inklusiven Kontexten. Die vorliegende Studie zeigt zum Teil starke positive, aber nicht signifikante Zusammenhänge mit Konstrukten aus dem Bereich pädagogischer Diagnostik. Im Bezug auf das Lernformat kann daher nur vorsichtig interpretiert werden, dass selbstgesteuertes Lernen dabei hilft, diesen Zusammenhang zu steigern. Daran anknüpfend stellt sich die generelle Frage zum Effekt der Kooperation von Lehrkräften im Kontext schulischer Inklusion. Meta-Analysen zu Effekten von Kooperation unter Lehrkräften im allgemeinen Schulbetrieb deuten auf eine Reduktion von Stress und Resignation hin (Vangrieken, Meredith, Packer und Kyndt 2017). In Anbetracht der Befürchtungen vieler Lehrkräfte bzgl. inklusiven Unterrichtens könnte der positive Effekt von Kooperation möglicherweise bereits in der Lehrkräftebildung aufgebaut werden. Denkbar ist etwa die Implementation didaktischer Modelle zur Zusammenarbeit von Lehramtsstudierenden mit an inklusionsorientierten Schulen tätigen Lehrkräften. Weitere empirische Forschung im Kontext inklusiven Unterrichtens bietet das Potenzial zu einer grundlegend evidenzbasierten Professionalisierung der Lehrkräftebildung.

\section{Literatur}

Allport, Gordon Willard. 1954. The nature of prejudice. Cambridge, Massachusetts: Addison-Wesley.

Amrhein, Bettina. 2011. Inklusion in der Sekundarstufe: Eine empirische Analyse. Bad Heilbrunn: Klinkhardt. 
Avramidis, Elias, Phil Bayliss und Robert Burden. 2002. »Inclusion in action: an in-depth case study of an effective inclusive secondary school in the south-west of England." International Journal of Inclusive Education 6, no.2: 143-163.

Avramidis, Elias, und Brahm Norwich. 2002. »Teachers' attitudes towards integration/inclusion: a review of the literature." European journal of special needs education 17, 2: 129-147.

Bakdash, Jonathan Z., und Laura R. Marusich. 2017. "Repeated measures correlation. "Frontiers in psychology 8:, 456 (April): 1-13.

Bakdash, Jonathan Z., und Laura R. Marusich. 2019. „Corrigendum: Repeated Measures Correlation.« Frontiers of Psychology 10, 1201 (Mai): 1-2.

Batsiou, Sofia, Evangelos Bebetsos, Panos Panteli und Panagiotis Antoniou. 2008. »Attitudes and intention of Greek and Cypriot primary education teachers towards teaching pupils with special educational needs in mainstream schools. «International Journal of Inclusive Education 12, 2: 201-219.

Baumert, Jürgen, und Mareike Kunter. 2006. „Stichwort: Professionelle Kompetenz von Lehrkräften.« Zeitschrift für Erziehungswissenschaft 9, 4: 469-520.

Baumert, Jürgen, und Mareike Kunter. 2011. „Das Kompetenzmodell von COACTIV. II Professionelle Kompetenzen von Lehrkräften: Ergebnisse des Forschungsprogramms COACTIV, herausgegeben von Mareike Kunter, Jürgen Baumert, Werner Blum, Uta Klusmann, Stefan Krauss und Michael Neubrand, 29-53. Münster, New York, München, Berlin: Waxmann.

Bland, J. Martin, und Douglas G. Altman. 1995. „Calculating correlation coefficients with repeated observations: part 1 Correlation within subjects.«BMJ 310: 446.

Bohner, Gerd, und Michaela Wänke. 2002. Attitudes and Attitude Change: Social Psychology. Hove, UK: Psychology Press.

Bromme, Rainer, Michael Jäger und Manfred Prenzel. 2014. »Empirische Bildungsforschung und evidenzbasierte Bildungspolitik.« Zeitschrift für Erziehungswissenschaft 17, 4: 3-54.

Bromme, Rainer, Manfred Prenzel und Michael Jäger. 2016. „Empirische Bildungsforschung und evidenzbasierte Bildungspolitik:Zum Zusammenhang von Wissenschaftskommunikation und Evidenzbasierung in der Bildungsforschung."Zeitschrift für Erziehungswissenschaft 19, 1: 129-146.

Brownell, Mary T., Alyson Adams, Paul Sindelar, Nancy Waldron und Stephanie Vanhover. 2006. »Learning from collaboration: The role of teacher qualities." Exceptional Children 72, no.2: 169-185.

Carpenter, Christopher J.. 2015. »A meta-analysis of the ELM's argument quality $\times$ processing type predictions." Human Communication Research 41, 4: 501-534.

Cohen, Jacob, Patricia Cohen, Stephen G. West und Leona S. Aiken. 2003. Applied multiple correlation/regression analysis for the social sciences. London: Routledge.

Darke, Peter R., Shelly Chaiken, Gerd Bohner, Sabine Einwiller, Hans-Peter Erb und J. Douglas Hazlewood. 1998. "Accuracy motivation, consensus information, and the law of large numbers: Effects on attitude judgment in the absence of argumentation."Personality and Social Psychology Bulletin 24, 11: 1205-1215.

Darling-Hammond, Linda. 2017. »Teacher education around the world: What can we learn from international practice?« European Journal of Teacher Education 40, 3: 291-309.

Davies, Philip. 1999. „What is evidence-based education? « British journal of educational studies 47, 2: 108-121.

De Boer, Anke, Sip Jan Pijl und Alexander Minnaert. 2011. »Regular primary schoolteachers' attitudes towards inclusive education: A review of the literature. «International journal of inclusive education 15, 3: 331-353.

Demirel, Melek, und Miray Dağyar. 2016. »Effects of Problem-Based Learning on Attitude: A Metaanalysis Study.«Eurasia Journal of Mathematics, Science \& Technology Education 12, 8:2115-2137. 
Eid, Michael, Mario Gollwitzer und Manfred Schmitt. 2011. Statistikund Forschungsmethoden. 2. Auflage. Weinheim: Beltz.

Ewing, Donna L., Jeremy J. Monsen und Stephan Kielblock. 2018. »Teachers' attitudes towards inclusive education: a critical review of published questionnaires." Educational Psychology in Practice 34, 2: 150-165.

Fischer, Christian, Henrike Kopmann, David Rott, Marcel Veber und Horst Zeinz. 2014. »Adaptive Lehrkompetenz und pädagogische Haltung. Lehrerbildung für eine inklusive Schule.« Jahrbuch für allgemeine Didaktik 4: 16-33.

Fleming, Monique A., und Richard E. Petty. 2000. »Identity and persuasion: An elaboration likelihood approach."In Applied social research. Attitudes, behavior, and social context: The role of norms and group membership, herausgegeben von D.J. Teddy und M. A. Hogg, 171-199. Mahwah, NJ, US: Lawrence Erlbaum Associates Publishers.

Ghanizadeh, Ahmad, Mohammad Jafar Bahredar und Seyed Reza Moeini. 2006. »Knowledge and attitudes towards attention deficit hyperactivity disorder among elementary school teachers." Patient Education and Counseling 63, 1-2: 84-88.

Gopnik, Alison, und Joshua B. Tenenbaum. 2007. „Bayesian networks, Bayesian learning and cognitive development."Developmental science 10, 3: 281-287.

Greiten, Silvia, Georg Geber, Annika Gruhn und Manuela Köninger. 2017. »Inklusion als Aufgabe für die Lehrerausbildung. Theoretische, institutionelle, curriculare und didaktische Herausforderungen für Hochschulen.« In Lehrerausbildung für Inklusion: Fragen und Konzepte zur Hochschulentwicklung, herausgegeben von Silvia Greiten, Georg Geber, Annika Gruhn und Manuela Köninger, 14-36. Münster: Waxmann.

Hamilton, Jennifer, Phillip E. Gagne und Gregory R. Hancock. 2003. »The Effect of Sample Size on Latent Growth Models.« Manuskript vorgestellt auf der Jahrestagung der American Educational Research Association, Chicago, 21.-25. April. Chicago: ERIC.

Hargreaves, Andy. 2000. »Four ages of professionalism and professional learning.« Teachers and Teaching 6, 2: 151-182.

Heinisch, Julia, Magdalena Sonnleitner und Astrid Rank. 2018. »Professionalisierung für Inklusionsituiertes Lernen in der universitären LehrerInnenbildung. « Jahrbuch Grundschulforschung 22: 296-302.

Hellmich, Frank und Gamze Görel. 2014. »Erklärungsfaktoren für Einstellungen von Lehrerinnen und Lehrern zum inklusiven Unterricht in der Grundschule.« Zeitschrift für Bildungsforschung 4, 3: 227-240.

Hillenbrand, Clemens, Conny Melzer und Junggyung Sung. 2014. »Lehrerbildung für Inklusion in Deutschland: Stand der Diskussion und praktische Konsequenzen." Theory and Practice of Education 19, 2: 147-171.

Kim, Ji-Ryun. 2011. »Influence of teacher preparation programmes on preservice teachers' attitudes toward inclusion. «International Journal of Inclusive Education 15, 3: 355-377.

Klug, Julia, Simone Bruder, Augustin Kelava, Christiane Spiel und Bernhard Schmitz. 2013. »Diagnostic competence of teachers: A process model that accounts for diagnosing learning behavior tested by means of a case scenario." Teaching and Teacher Education 30: 38-46.

Klug, Julia, Simone Bruder und Bernhard Schmitz. 2016. „Which variables predict teachers diagnostic competence when diagnosing students' learning behavior at different stages of a teacher's career?« Teachers and Teaching 22, no.4: 461-484.

König, Johannes, Kristina Gerhard, Kai Kaspar und Conny Melzer. 2019. »Professionelles Wissen von Lehrkräften zur Inklusion: Überlegungen zur Modellierung und Erfassung mithilfe standardisierter Testinstrumente."Pädagogische Rundschau 73, 1:43-64.

Kultusministerkonferenz (KMK) und Hochschulrektorenkonferenz (HRK) (2015). Lehrerbildung 
für eine Schule der Vielfalt. Gemeinsame Empfehlung von Hochschulrektorenkonferenz und Kultusministerkonferenz. https://www.kmk.org/fileadmin/Dateien/ veroeffentlichungen_beschluesse/2015/2015_03_12-Schule-der-Vielfalt.pdf.

Kurniawati, Farida, Anke A. De Boer, Alexander E. M. G. Minnaert und Frieda Mangunsong. 2014. »Characteristics of primary teacher training programmes on inclusion: a literature focus. «Educational Research 56, 3: 310-326.

Kurniawati, Farida, Anke A. De Boer, Alexander E. M. G. Minnaert und Frieda Mangunsong. 2017. »Evaluating the effect of a teacher training programme on the primary teachers' attitudes, knowledge and teaching strategies regarding special educational needs." Educational Psychology 37, 3: 287-297.

Kuvac, Meltem, und Isil Koc. 2018. »The effect of problem-based learning on the metacognitive awareness of pre-service science teachers."Educational Studies 21, 1: 1-21.

Lavine, Howard, und Mark Snyder. 1996. „Cognitive processing and the functional matching effect in persuasion: The mediating role of subjective perceptions of message quality." Journal of Experimental Social Psychology 32, 6: 580-604.

Leipziger, Eva, Tobias Tretter und Markus Gebhardt. 2012. »Inklusion an oberfränkischen Grundschulen."Zeitschrift für Heilpädagogik 10: 433-439.

Lindmeier, Christian. 2014. »Aktuelle bildungspolitische Bemühungen um eine inklusionsorientierte Erneuerung der deutschen Lehrerausbildung."Zeitschrift für Heilpädagogik 3: 84-97.

Liu, Yingna, Jialing Li, Qiaolan Zheng, Charles M. Zaroff, Brian J. Hall, Xiuhong Li und Yuantao Hao. 2016. "Knowledge, attitudes, and perceptions of autism spectrum disorder in a stratified sampling of preschool teachers in China."BMC psychiatry 16, 1: 142.

Marsh, Herbert W., und Rhonda G. Craven. 2006. »Reciprocal effects of self-concept and performance from a multidimensional perspective: Beyond seductive pleasure and unidimensional perspectives."Perspectives on psychological science 1, 2: 133-163.

Marx, Christian, Annika Goeze, Thamar Voss, Verena Hoehne, Viola Katharina Klotz und Josef Schrader. (2017). "Pädagogisch-psychologisches Wissen von Lehrkräften aus Schule und Erwachsenenbildung: Entwicklung und Erprobung eines Testinstruments.« Zeitschrift für Erziehungswissenschaft 20, 1: 165-200.

Mulholland, Sarah. 2016. »ADHD-specific knowledge and attitudes of teachers (ASKAT): Development and validation of a new research instrument." International Journal of Educational Research 77: 109-116.

Mulholland, Sarah M., Therese M. Cumming und Jae Yup Jung. 2015. „Teacher attitudes towards students who exhibit ADHD-type behaviours. «Australasian Journal of Special Education 39, 1: $15-36$.

Mullen, Edward J. 2016. »Reconsidering the sidea of evidence in evidence-based policy and practice." European journal of social work 19, 3-4: 310-335.

OECD (2014). TALIS 2014 Results. An International Perspective on Teaching und Learning, TALIS, OECD Publishing.

Ohle, Annika, Nele McElvany, Holger Horz und Mark Ullrich. 2015. »Text-picture integration-Teachers' attitudes, motivation and self-related cognitions in diagnostics." Journal for educational research online 7, 2: 11-33.

Petty, Richard E., und John T. Cacioppo. 1986. »The elaboration likelihood model of persuasion." Advances in Experimental Social Psychology 19: 123-205.

Petty, Richard E., und Duane T. Wegener. 1998. »Matching versus mismatching attitude functions: Implications for scrutiny of persuasive messages." Personality and Social Psychology Bulletin 24, 3: 227-240.

Rasch, Dieter, und Dieter Schott. 2018. Mathematical Statistics. Oxford: Wiley. 
Retelsdorf, Jan, Johannes Bauer, S. Kristina Gebauer, Tabea Kauper und Jens Möller. 2014. „Erfassung berufsbezogener Selbstkonzepte von angehenden Lehrkräften (ERBSE-L).»Diagnostica 60: 98-110.

Rey, Thomas, Hendrik Lohse-Bossenz, Albrecht Wacker und Vera Heyl. 2018. »Adaptive Planungskompetenz bei angehenden Lehrkräften in der zweiten Phase der Lehrerbildung. Befunde einer Pilotierungsstudie aus Baden-Württemberg.« heiEDUCATION Journal. Transdisziplinäre Studien zur Lehrerbildung 1-2: 127-150.

Sackett, David L., William MC Rosenberg, JA Muir Gray, R. Brian Haynes und W. Scott Richardson. 1996. »Evidence based medicine: what it is and what it isn't.«BMJ 312: 71-72.

Santagata, Rossella, und Cathery Yeh. 2016. „The role of perception, interpretation, and decision making in the development of beginning teachers' competence." ZDM 48, 1-2: 153-165.

Savage, Robert Samuel, und Ozlem Erten. 2015. „Teaching in Inclusive Classrooms: The Link Between Teachers Attitudes-Practices and Student Outcomes." Journal of Psychology \& Psychotherapy 5, 6: 1-7.

Schum, David. 2012. „Classifying forms and combinations of evidence: Necessary in a science of evidence.« In Evidence, inference and enquiry, herausgegeben von P. Dawid, W. Twining und D. Vasilaki, 11-36. New York, NY: Oxford University Press.

Schürer, Sina, Stefanie van Ophuysen und Bea Bloh. 2018. »Inklusion und Schulqualität-Welche Erwartungen haben Lehramtsstudierende an die Konsequenzen schulischer Inklusion?« In Profession und Disziplin, herausgegeben von Susanne Miller, Birgit Holler-Nowitzki, Brigitte Kottmann. Svenja Lesemann, Birte Letmathe-Henkel, Nikolas Meyer, René Schroeder und Katrin Velten, 289-295. Wiesbaden: Springer VS.

Schwab, Susanne, und Susanne Seifert. 2015. „Einstellungen von Lehramtsstudierenden und Pädagogikstudierenden zur schulischen Inklusion-Ergebnisse einer quantitativen Untersuchung.« Zeitschrift für Bildungsforschung 5, 1: 73-87.

Seaman, Samuel L., James Algina und Stephen F. Olejnik. 1985. »Type I error probabilities and power of the rank and parametric ANCOVA procedures." Journal of Educational Statistics 10, 4: 345-367.

Seifried, Stefanie, und Vera Heyl. 2016. „Konstruktion und Validierung eines Einstellungsfragebogens zu Inklusion für Lehrkräfte (EFI-L).« Empirische Sonderpädagogik 1: 22-35.

Spiel, Christiane, und Dagmar Strohmeier 2012. »Evidence-based practice and policy: When researchers, policy makers, and practitioners learn how to work together." European Journal of Developmental Psychology 9, 1: 150-162.

Sermier Dessemontet, Rachel, Valérie Benoit und Gérard Bless. 2011. „Schulische Integration von Kindern mit einer geistigen Behinderung. Untersuchung der Entwicklung der Schulleistungen und der adaptiven Fähigkeiten, der Wirkung auf die Lernentwicklung der Mitschüler sowie der Lehrereinstellungen zur Integration. Empirische Sonderpädagogik 3, 4: 291-307.

Syring, Marcus, Teresa Tillmann, Sabine Weiß und Ewald Kiel. 2018. »Positive Einstellung zur Inklusion - ablehnende Haltung zur Umsetzung in der Schule: Analyse des Widerspruchs durch Überprüfung eines aus der Heterogenitätsforschung adaptierten Messinstruments für die Inklusion an Lehramtsstudierenden."Psychologie in Erziehung und Unterricht 65, 3: 206-220.

Van Mieghem, Aster, Karine Verschueren, Katja Petry und Elke Struyf. 2018. »An analysis of research on inclusive education: a systematic search and meta review. International Journal of Inclusive Education 15, 1: 1-15.

Vangrieken, Katrien, Chloé Meredith, Tlalit Packer und Eva Kyndt. 2017. »Teacher communities as a context for professional development: A systematic review. Teaching and Teacher Education 61: 47-59. 
Voss, Thamar, Olga Kunina-Habenicht, Verena Hoehne und Mareike Kunter. 2015. "Stichwort Pädagogisches Wissen von Lehrkräften: Empirische Zugänge und Befunde.« Zeitschrift für Erziehungswissenschaft 18, 2: 187-223.

Wedel, Alexander, und Christin R. Müller. 2016. »Test zum pädagogischen Diagnosewissen von Lehrkräften (PDW)« Unveröffentlichtes Manuskript. Berlin: TU Berlin.

Wedel, Alexander, und Christin R. Müller. 2017. „Skala zur Erfassung des diagnostischen Selbstkonzepts von Lehrkräften (LSD)« Unveröffentlichtes Manuskript. Berlin: TU Berlin.

Wedel, Alexander, Müller, Christin R., Pfetsch, Jan und Angela Ittel. 2019. »Entwicklung diagnostischer Kompetenz in der Lehramtsausbildung - Effekte problemorientierten Lernens mit textbasierten Fällen. «In Evidenzbasierung in der Lehrkräftebildung. Edition Zeitschrift für Erziehungswissenschaft Band 4, hrsg. v. Ingrid Gogolin, Bettina Hannover und Annette Scheunpflug, 95-122. Wiesbaden: Springer VS.

Wegener, Duane T., und Richard E. Petty. 1998. »The naive scientist revisited: Naive theories and social judgment." Social Cognition 16, 1: 1-7.

Wenden, Anita. 1998. Learner Strategies for Learner Autonomy. Great Britain: Prentice Hall.

Winter, Stephan, und Nicole C. Krämer. 2016. „Who's right: The author or the audience? Effects of user comments and ratings on the perception of online science articles." Communications 3: 339-360.

Winter, Stephan, Nicole C. Krämer, Leonie Rösner und German Neubaum. 2015. »Don't keep it (too) simple: How textual representations of scientific uncertainty affect laypersons' attitudes." Journal of Language and Social Psychology 34, 3: 251-272.

Youssef, Marsha K., Gerard Hutchinson und Farid F. Youssef. 2015. »Knowledge of and attitudes toward ADHD among teachers insights from a Caribbean Nation.« SAGE Open 5: 1-8.

\section{Die Autorlnnen}

Alexander Wedel ist wissenschaftlicher Mitarbeiter im Fachgebiet Pädagogische Psychologie der Technischen Universität Berlin. Er forscht vor dem Hintergrund erziehungswissenschaftlicher, psychologischer und soziologischer Theorien zu Lernen und Bildung in formellen und informellen Kontexten. Aktuelle Forschungsthemen sind Diagnostische Kompetenz von Lehrkräften und Lehramtsstudierenden, Instructional Design und Unterrichtsaufgaben sowie Empathie und personenbezogene Informationsverarbeitung. Darüber hinaus liegen Arbeitsschwerpunkte in biografischen Bildungsprozessen über die Lebensspanne sowie Männlicher Sozialisation und Lebensbewältigung.

Kontakt: alexander.wedel@tu-berlin.de

Jan Pfetsch ist Gastprofessor und stellvertretender Leiter des Fachgebiets Pädagogische Psychologie am Institut für Erziehungswissenschaft der Technischen Universität Berlin. Seine Forschungsschwerpunkte liegen in pädagogisch-psychologischen, entwicklungspsychologischen und sozialpsychologischen Themenfeldern. So forscht er zu Empathie im Online- und OfflineKontext, Medienkompetenz, diagnostischer Kompetenz von Lehramtsstudierenden, Lernen mit digitalen Medien sowie aggressivem und prosozialem Verhalten bei Kindern und Jugendlichen. Momentan leitet er Forschungsprojekte im Bereich Lehrkräftebildung, Digitalisierung in der Hochschullehre sowie Berufsinteressen für MINT Fächer (Mathematik, Informatik, Naturwissenschaften, Technik).

Kontakt: jan.pfetsch@tu-berlin.de 
Angela Ittel ist Professorin für Pädagogische Psycholgie am Institut für Erziehungswissenschaft an der Technischen Universität Berlin. Ihre Forschungsinteressen umfassen folgende Themenschwerpunkte: Geschlechstspezifische Interessensentwicklung und Berufswahlorientierung, Entwicklungsbedingungen der Heterogenität im Jugendalter und jungen Erwachsenenalter. Seit 2014 ist sie Vizeipräsidentin der Technischen Universität Berlin und verantwortet die Bereeich Strategische Entwicklung, Nachwuchs und Lehrkräftebildung. DFG Forschung, Diversität, Gleichstellung und Internationalisierung sind Schwerpunkte und Bereich der Strategischen Entwicklung.

Kontakt: angela.ittel@tu-berlin.de 